\title{
ON COMMUTATOR LAWS IN GROUPS
}

\author{
I. D. MACDONALD and B. H. NEUMANN
}

(Received 25 April 1987)

Communicated by J. F. Price

Dedicated to Robert Edwards in recognition of

25 years' distinguished contribution to mathematics in Australia, on the occasion of his retirement

\begin{abstract}
There are some well-known laws that the commutator satisfies in groups, and that go by some or all of the names Jacobi, Witt, Hall; and there are also some lesser-known laws. This is an attempt at an axiomatic study of the interdependence and independence of these laws.

1980 Mathematics subject classification (Amer. Math. Soc.) (1985 Revision): primary 20 A 05; secondary 08 A 99.
\end{abstract}

\section{Introduction}

Luise-Charlotte Kappe had some time ago asked one of us (IDM) whether there are any commutator laws in 4 variables that do not "follow" from those in 3 variables and that are valid in all groups. The present investigation has arisen out of an attempt ot answer this question. We try here to find out something about the logical interdependence of the various commutator laws that are known. Forming the commutator can be looked upon as a binary operation; as we wish to study the laws this operation satisfies, and how they imply or do not imply each other, we introduce a symbol, $\kappa$ (no pun intended), for this operation. We write it as a right-hand operator: $x y \kappa$, and we call the result a " $\kappa$-tator". As we are working in groups, we need the group operations, too. These should, for the sake of consistency, also be denoted by lower case Greek letters and written

(C) 1988 Australian Mathematical Society 0263-6115/88 \$A2.00+0.00 
as right-hand operators. However, we instead adopt a hybrid system, using the usual group operation symbols: $x \cdot y$ for the product, $x^{-1}$ for the inverse, $e$ for the unit element. (Note that the dot - will always be used for multiplication, not just juxtaposition.) The "real" commutator is

$$
[x, y]:=x^{-1} \cdot y^{-1} \cdot x \cdot y .
$$

The notation for left-normed higher commutators is conventional:

$$
[x, y, z]:=[[x, y], z] .
$$

We also use the usual notation for conjugation

$$
x^{y}:=y^{-1} \cdot x \cdot y, \quad x^{-y}:=y^{-1} \cdot x^{-1} \cdot y .
$$

A number of laws involving the commutator are well known. Some, like

$$
[x, e]=e,
$$

are trivial. Others, like

$$
[x \cdot y, z]=[x, z]^{y} \cdot[y, z],
$$

are immediately verified, but will in the sequel be looked at more closely. Then there are the laws that go by several of the names Jacobi, Witt, Hall. There is no unanimity about what they are; probably the best-known is

$$
\left[x, y^{-1}, z\right]^{y} \cdot\left[y, z^{-1}, x\right]^{z} \cdot\left[z, x^{-1}, y\right]^{x}=e,
$$

which is certainly not due to Jacobi, whose concern was what is now called Lie rings; nor is it due, as far as we have been able to find out, to either Ernst Witt or Philip Hall in this form. There are a number of such laws, almost all of the form

$$
w(x, y, z) \cdot w(y, z, x) \cdot w(z, x, y)=e,
$$

where $w$ is some commutator word; and there are corresponding laws in four or more variables. Some of them will be described in the next section. It is their interdependence or independence that we wish to study. The algebraic systems we consider will be called "kappa-groups".

The first author records his thanks to David W. Rosenthal for stimulating discussions.

\section{The laws}

We group the laws we want to consider into several sets; the first set contains a single law, which will never be assumed, and an equivalent:

$$
x y \kappa=[x, y] \text {. }
$$


This is equivalent to

$$
x \cdot x y \kappa=x^{y} .
$$

The second set consists of the "trivial" laws which will always be assumed:

$$
\begin{aligned}
x e \kappa & =e, \\
e x \kappa & =e, \\
x x \kappa & =e, \\
x y \kappa \cdot y x \kappa & =e,
\end{aligned}
$$

or, equivalently,

$$
y x \kappa=(x y \kappa)^{-1} .
$$

The third set consists of laws whose counterparts (that is to say the laws that result by replacing $x y \kappa$ by $[x, y]$ ) are not satisfied in all groups, but that will sometimes be used

$$
\begin{gathered}
x y \kappa=e, \\
x y \kappa z \kappa=e, \\
x y \kappa z t \kappa^{2}=e, \\
(x \cdot y) z \kappa=x z \kappa \cdot y z \kappa, \\
x(y \cdot z) \kappa=x y \kappa \cdot x z \kappa .
\end{gathered}
$$

A kappa-group in which $S 1$ is satisfied will be called "kappa-abelian", one in which S2 is satisfied "kappa-nilpotent of class 2", one in which S3 is satisfied "kappa-metabelian", and so on. If S4 is satisfied, we shall call kappa "left linear"; and "bilinear" if both S4 and S5 are satisfied. In a nilpotent group of class 2, the commutator is bilinear, and conversely, but for $\kappa$-tators this is not always the case.

The fourth and fifth sets are more important: they consist of those whose counterparts are satisfied in all groups (except for J8 and J9, which require the kappa-group to be kappa-metabelian), and whose interdependence or independence will be investigated.

$$
\begin{gathered}
x y \kappa z \kappa=[x y \kappa, z], \\
(x \cdot y) z \kappa=x z \kappa \cdot x z \kappa y \kappa \cdot y z \kappa, \\
x(y \cdot z) \kappa=x z \kappa \cdot x y \kappa \cdot x y \kappa z \kappa, \\
x \cdot y \cdot(x \cdot y) z \kappa=x \cdot x z \kappa \cdot y \cdot y z \kappa, \\
x \cdot x(y \cdot z) \kappa=x \cdot x y \kappa \cdot(x \cdot x y \kappa) z \kappa, \\
z^{-1} \cdot x y \kappa \cdot z=\left(z^{-1} \cdot x \cdot z\right)\left(z^{-1} \cdot y \cdot z\right) \kappa, \\
x y \kappa \cdot x y \kappa z \kappa=(x \cdot x z \kappa)(y \cdot y z \kappa) \kappa, \\
x y^{-1} \kappa=x y \kappa^{-1}
\end{gathered}
$$




$$
\begin{gathered}
x y^{-1} \kappa=y x \kappa \cdot y x \kappa y^{-1} \kappa, \\
x^{-1} y \kappa=x y \kappa^{-x^{-1}}, \\
x^{-1} y \kappa=y x \kappa \cdot y x \kappa x^{-1} \kappa .
\end{gathered}
$$

I1 says that if the first argument in a $\kappa$-tator is itself a $\kappa$-tator, then the whole $\kappa$-tator coincides with the commutator; and it is easy to see that because of A4, the same is then true also if the second argument of the outside $\kappa$-tator is itself a $\kappa$-tator. I 2 and I 3 give the connection between multiplication and $\kappa$-tation. The next four laws deal with the connection between $\kappa$-tation and conjugation in various ways. In $\mathrm{I} 4$ and $\mathrm{I} 5$, the first factor $x$ can, of course, be cancelled on both sides; this we have refrained from doing in order to show more clearly that these laws are a translation of the "index laws" of conjugation. The last four express the connection between $\kappa$-tation and inversion; I9 and I11 can be applied to themselves again and again, ad infinitum. The next set of laws consists of the Jacobi-Witt-Hall type laws; they are all of the form

$$
w(x, y, z) \cdot w(y, z, x) \cdot w(z, x, y)=e
$$

if they involve 3 variables, or

$$
w(x, y, z, t) \cdot w(y, z, t, x) \cdot w(z, t, x, y) \cdot w(t, x, y, z)=e
$$

if they involve 4 variables (and analogously for more than 4 variables-the corresponding laws in 5 and more variables will not enter this discussion). We list only the words $w$,

$$
\begin{gathered}
w(x, y, z)=x y^{-1} \kappa z \kappa \cdot x y^{-1} \kappa z \kappa y \kappa, \\
w(x, y, z)=(y x \kappa)(z \cdot z y \kappa) \kappa, \\
w(x, y, z)=(x \cdot y) z \kappa, \\
w(x, y, z)=x z \kappa \cdot x z \kappa y \kappa \cdot y z \kappa, \\
w(x, y, z, t)=(x \cdot y \cdot z) t \kappa, \\
w(x, y, z, t)=x t \kappa \cdot x t \kappa y \kappa \cdot y t \kappa \cdot(x t \kappa \cdot x t \kappa y \kappa \cdot y t \kappa) z \kappa \cdot z t \kappa .
\end{gathered}
$$

The first of these is a translation of the best-known form of the Jacobi-Witt-Hall identity or identities; so is the second. The commutator analogues of J3 and J5 first occurred in the (unpublished part of the) Cambridge PhD thesis of one of us (BHN); J4 and J6 are essentially the same as J3 and J5, respectively, but making use of $\mathrm{I} 2$, which will only rarely be valid in the kappa-groups we shall later exhibit. J6 could be further expanded by repeatedly using 12, but we refrain from making it even more complicated. There are, however, two more laws that arise from J3 and J5 and that we wish to look at; they are formed in the following way: In the commutator analogue of $\mathrm{J} 3$ (or J5), collect all commutators of weight 2 to 
the left until they cancel; this introduces commutators of higher weight, making a long and complicated product, in which we replace commutators by kappas. This gives

$$
\begin{aligned}
x z \kappa y \kappa & \cdot x z \kappa y \kappa z x \kappa^{2} \cdot y z \kappa z x \kappa^{2} \cdot y z \kappa z x \kappa^{2} z y \kappa^{2} \cdot y x \kappa z x \kappa^{2} \\
& \cdot y x \kappa z x \kappa^{2} z y \kappa^{2} \cdot y x \kappa z x \kappa^{2} x y \kappa^{2} \cdot y x \kappa z x \kappa^{2} x y \kappa^{2} z y \kappa^{2} \cdot y x \kappa z \kappa \\
& \cdot y x \kappa z \kappa z y \kappa^{2} \cdot y x \kappa z \kappa x y \kappa^{2} \cdot y x \kappa z \kappa x y \kappa^{2} z y \kappa^{2} \cdot y x \kappa z \kappa z x \kappa^{2} \\
& \cdot y x \kappa z \kappa z x \kappa^{2} z y \kappa^{2} \cdot y x \kappa z \kappa z x \kappa^{2} x y \kappa^{2} \cdot y x \kappa z \kappa z x \kappa^{2} x y \kappa^{2} z y \kappa^{2} \\
& \cdot z y \kappa x y \kappa^{2} \cdot z y \kappa x \kappa \cdot z y \kappa x \kappa x y \kappa^{2}=e .
\end{aligned}
$$

This is, of course, a quite unmanageable expression, but in a kappa-metabelian kappa-group, that is to say under the assumption that $\mathrm{S3}$ is satisfied, it simplifies considerably, namely to

$$
x z \kappa y \kappa \cdot y x \kappa z \kappa \cdot z y \kappa x \kappa=e .
$$

We refrain from writing the law that corresponds to 36 out in full; in a kappametabelian kappa-group it again simplifies, and becomes, with the same convention as in $\mathrm{J} 5$ and $\mathrm{J} 6$,

$$
w(x, y, z, t)=x t \kappa y \kappa \cdot x t \kappa z \kappa \cdot x t \kappa y \kappa z \kappa \cdot y t \kappa z \kappa .
$$

\section{Some positive results}

Here we list some interdependences of the various laws (not by any means all of them: there are too many). Recall that we assume kappa to satisfy the laws A1-4: they will be used without further mention. They are not independent: it is easily seen that A1 and A4 together imply A2, and A2 and A4 together imply A1.

LEMMA 3.1. Of I1, I2, I4, any two imply the third.

ProOF. Assume I1 and I2. Observe that I4 can be rewritten in the form

$$
(x \cdot y) z \kappa=y^{-1} \cdot x z \kappa \cdot y \cdot y z \kappa,
$$

with the same left-hand side as I2. Now use I1 to rewrite the middle $\kappa$-tator on the right-hand side of 12 , to get

$$
(x \cdot y) z \kappa=x z \kappa \cdot(z x \kappa)^{-1} \cdot y^{-1} \cdot x z \kappa \cdot y \cdot y z \kappa,
$$

which, after cancellation on the right-hand side, gives 3.11 , and thus 14 . To derive $\mathrm{I} 2$ from $\mathrm{I} 1$ and I4, the same steps can be reversed. Finally assume I2 and I4, the latter in the equivalent form 3.12. As the left-hand sides are equal, we 
equate the right-hand sides, then cancel the first and last factors to get I1 (with $y$ and $z$ interchanged).

LEMMA 3.2. I4 and I5 together are equivalent to I1, I2, and I3 together.

PROOF. Begin with I5 and apply 14 to the right-hand side, with $x y \kappa$ substituted for $y$. This gives

$$
x \cdot x(y \cdot z) \kappa=x \cdot x z \kappa \cdot x y \kappa \cdot x y \kappa z \kappa,
$$

which, after cancelling the factor $x$ on the left, becomes I3. Now use 3.11, which is equivalent to I4, and invert both sides to get

$$
z(x \cdot y) \kappa=z y \kappa \cdot y^{-1} \cdot z x \kappa \cdot y,
$$

where we have used A4 repeatedly. Changing $x$ to $y, y$ to $z, z$ to $x$ gives

$$
x(y \cdot z) \kappa=x z \kappa \cdot z^{-1} \cdot x y \kappa \cdot z,
$$

and comparison with $\mathrm{I} 3$ then leads to

$$
x y \kappa \cdot x y \kappa z \kappa=z^{-1} \cdot x y \kappa \cdot z
$$

which finally, after dividing by $x y \kappa$ on the left, becomes I1. Now I2 follows from Lemma 3.1. All the steps are reversible, to give the converse (in fact only I5 needs to be derived, as the validity of I4 follows from Lemma 3.1).

LEMMA 3.3. J3 and J5 are equivalent.

ProOF. To derive $\mathrm{J} 3$ from J5, put one of the variables, say $t$, equal to $e$. For the converse, replace $z$ in $\mathrm{J} 3$ by $u \cdot v$, say. This gives

$$
(x \cdot y)(u \cdot v) \kappa \cdot(y \cdot u \cdot v) x \kappa \cdot(u \cdot v \cdot x) y \kappa=e .
$$

In this, interchange $x$ with $u$ and $y$ with $v$, to get

$$
(u \cdot v)(x \cdot y) \kappa \cdot(v \cdot x \cdot y) u \kappa \cdot(x \cdot y \cdot u) v \kappa=e .
$$

Transform the two sides of 3.31 by $(x \cdot y)(u \cdot v) \kappa$, then multiply the left-hand side of 3.31 (thus transformed) from the left into the left-hand side of 3.32 . The result is, but for the names of the variables, J5.

\section{Independence results}

To show the independence of certain of the above laws from some of the others, we consider models of kappa-groups. For example, to show that N1 is independent of all the rest, we take a strictly non-abelian group, say the 
symmetric group of degree 3 , and we define $\kappa$ as trivial, that is to say so that it satisfied S1, and the kappa-group becomes kappa-abelian: it is easy to see that in a kappa-abelian kappa-group all laws $\mathrm{A} n, \mathrm{~S} n, \mathrm{I} n, \mathrm{~J} n$ are trivially satisfied.

Ideally there should be to every law a model which fails to satisfy that law, but satisfied all the others; but this is too much to hope for, as we already know that some of the laws or groups of laws imply others. In any case we have far too many laws in our list to be able to survey their interdependences and independences completely.

For the present our aim is very much more modest: we shall, out of a considerable number we have investigated, present just 3 , with a list of the laws they satisfy and those they fail to satisfy: we call this their "profile".

Our first model is quite small: the underlying group is the 4-group. As a group it is generated by two elements, say $a$ and $b$, which satisfy the (defining) relations

$$
a^{2}=b^{2}=[a, b]=e
$$

We define $\kappa$ by

$$
x y \kappa=a \text { unless } x=e \text { or } y=e \text { or } x=y .
$$

The computations in this kappa-group, which we call M1, are not difficult, but we omit them and just state the result.

THEOREM 4.1. The kappa-group M1 satisfies S3, S4, S5, I4, I6-8, I10, and $\mathrm{J} 2-9$; it fails to satisfy $\mathrm{S} 1, \mathrm{~S} 2, \mathrm{I} 1-3, \mathrm{I} 5, \mathrm{I} 9, \mathrm{I} 11$, and $\mathrm{J} 1$.

This model illustrates the fact that $\kappa$ can be bilinear without the kappa-group being kappa-nilpotent of class 2 (M1 is, in fact, not kappa-nilpotent of any class). It also shows that $\mathrm{I} 4$ by itself does not imply any of $\mathrm{I} 1, \mathrm{I} 2$, or $\mathrm{I} 3$.

The second model, which we denote by $\mathrm{M} 2$, has as its underlying group the direct product of a free nilpotent-of-class-2 group of rank 2 with an infinite cyclic group. We generate it by four elements $a, b, c, d$ with the defining relations,

$$
[a, b]=c, \quad[a, c]=[a, d]=[b, c]=[b, d]=[c, d]=e .
$$

If

$$
x=a^{x_{1}} \cdot b^{x_{2}} \cdot c^{x_{3}} \cdot d^{x_{4}}
$$

and

$$
y=a^{y_{1}} \cdot b^{y_{2}} \cdot c^{y_{3}} \cdot d^{y_{4}}
$$


we put

$$
x y \kappa=c^{x_{2} \cdot y_{1}-x_{1} \cdot y_{2}} \cdot d^{u_{12}+x_{3} \cdot y_{1}-x_{1} \cdot y_{3}}
$$

where

$$
u_{12}=x_{2} \cdot y_{1} \cdot\left(y_{1}-1\right) / 2-x_{1} \cdot\left(x_{1}-1\right) \cdot y_{2} / 2 \text {. }
$$

This makes M2 kappa-nilpotent of class (strictly) 3. Here the verification of the profile is quite laborious; we omit it, but just state the result:

THEOREM 4.2. The kappa-group M2 satisfies S3, I2, I3, I5, I7, J1-J9, and fails to satisfy S1, S2, S4, S5, I1, I4, I6, I8-I11.

This model shows, inter alia, that 12 and 13 together do not imply I1, nor I4. The model can be modified by giving $a, b, c, d$ finite order, say $p$, so that the whole kappa-group becomes finite of order $p^{4}$.

The last model we exhibit, M3, has as underlying group the free abelian group of rank 10 . We write the elements as vectors and the $i$ th component of an element $x$ as $x_{i}$, where $i$ ranges from 1 to 10 , and $x_{i}$ is an integer; the group operation is (vector) addition, and kappa is defined by

$$
\begin{gathered}
(x y \kappa)_{i}=0 \text { for } i=1,2,3,4, \\
(x y \kappa)_{5}=x_{1} \cdot y_{2}-x_{2} \cdot y_{1}, \\
(x y \kappa)_{6}=x_{2} \cdot y_{3}-x_{3} \cdot y_{2}, \\
(x y \kappa)_{7}=x_{3} \cdot y_{4}-x_{4} \cdot y_{3} \\
(x y \kappa)_{8}=x_{1} \cdot y_{6}-x_{6} \cdot y_{1}+x_{5} \cdot y_{3}-x_{3} \cdot y_{5}, \\
(x y \kappa)_{9}=x_{2} \cdot y_{7}-x_{7} \cdot y_{2}+x_{6} \cdot y_{4}-x_{4} \cdot y_{6} \\
(x y \kappa)_{10}=x_{1} \cdot y_{9}-x_{9} \cdot y_{1}+x_{5} \cdot y_{7}-x_{7} \cdot y_{5}+x_{8} \cdot y_{4}-x_{4} \cdot y_{8} .
\end{gathered}
$$

Again the checking of the various laws is laborious and not very interesting, and we omit the details, but just state the result:

THEOREM 4.3. The profile of $\mathrm{M} 3$ is as follows: S4, S5, I4, I6, J3-5, J8 are satisfied, and $\mathrm{S} 1, \mathrm{~S} 2, \mathrm{~S} 3, \mathrm{I} 1-3, \mathrm{I} 5,17-11, \mathrm{~J} 1, \mathrm{~J} 2, \mathrm{~J} 6, \mathrm{~J} 7, \mathrm{~J} 9$ are not.

This model shows that $\mathrm{J} 6$ and $\mathrm{J9}$ do not follow from J3, J4, J5, J8, and thus at least partially answers Luise-Charlotte Kappe's question; however, it is not yet the answer we are aiming at, as the model also fails to satisfy J7. We have at various times thought that we could make models that satisfied $\mathrm{J} 7$ but not $\mathrm{J} 9$, nor the long and elaborate law in 4 variables that corresponds to $\mathrm{J} 5$ in the way $\mathrm{J} 7$ corresponds to $\mathrm{J} 3$, and that we have refrained from writing down, but careful checking has always disappointed our hopes. 
We conclude by tabulating, for comparison, the profiles of the three models of kappa-groups we have exhibited

$\begin{array}{rrrrrrrrrrrrrrrrr} & & & S & & & & & & & I & & & \\ & 1 & 2 & 3 & 4 & 5 & 1 & 2 & 3 & 4 & 5 & 6 & 7 & 8 & 9 & 10 & 11 \\ \text { M1 } & 0 & 0 & 1 & 1 & 1 & 0 & 0 & 0 & 1 & 0 & 1 & 1 & 1 & 0 & 1 & 0 \\ \text { M2 } & 0 & 0 & 1 & 0 & 0 & 0 & 1 & 1 & 0 & 1 & 0 & 1 & 0 & 0 & 0 & 0 \\ \text { M3 } & 0 & 0 & 0 & 1 & 1 & 0 & 0 & 0 & 1 & 0 & 1 & 0 & 0 & 0 & 0 & 0\end{array}$

\begin{tabular}{|c|c|c|c|c|c|c|c|c|}
\hline & & & & & $J$ & & & \\
\hline & 1 & 1 & 3 & 4 & 5 & 6 & 7 & 8 \\
\hline M1 & 0 & 1 & 1 & 1 & 1 & 1 & 1 & 1 \\
\hline M2 & 1 & 1 & 1 & 1 & 1 & 1 & 1 & 1 \\
\hline M3 & 0 & 0 & 1 & 1 & 1 & 0 & 0 & 1 \\
\hline
\end{tabular}

-(the entries are, of course, the boolean values, that is to say, a 1 means that the law is satisfied, a 0 that it is not satisfied, in the model).

Department of Mathematics

Lafayette College

Easton, Pennsylvania 18042

U.S.A.
Department of Mathematics Institute of Advanced Studies Australian National University Canberra, ACT 2601 Australia and

Division of Mathematics and Statistics Commonwealth Scientific and Industrial Research Organization Canberra, ACT 2601 Australia 